\title{
Nachhaltige Entwicklung
}

\section{- die notwendige Utopie}

Le développement durable: l'utopie nécessaire

\section{Ernst A. Brugger}

\section{(2) OpenEdition}

12 Journals

Electronic version

URL: http://journals.openedition.org/sjep/1246

DOI: 10.4000/sjep.1246

ISSN: 1663-9677

Publisher

Institut de hautes études internationales et du développement

\section{Printed version}

Date of publication: 1 février 1993

Number of pages: 207-214

ISSN: 1660-5926

\section{Electronic reference}

Ernst A. Brugger, « Nachhaltige Entwicklung

- die notwendige Utopie », Schweizerisches Jahrbuch für Entwicklungspolitik [Online], 12 | 1993, Online erschienen am: 30 April 2013, abgerufen am 08 September 2020. URL : http://

journals.openedition.org/sjep/1246 ; DOI : https://doi.org/10.4000/sjep.1246 


\title{
Nachhaltige Entwicklung - die notwendige Utopie*
}

\author{
Ernst A. Brugger
}

\section{1. "Rio"-Die Wende?}

"Nach Rio ist die Welt anders". Dies ist die Kurzbilanz von Maurice Strong, Generalsekretär der UNO-Konferenz über Umwelt und Entwicklung (UNCED) vom Juni 1992 in Rio (1). Stimmt diese optimistische Einschätzung oder vielmehr die Meinung der Pessimisten, dass nach Rio der freie Fall von hoher Rhetorik zu tiefem Realisierungsniveau droht? Beides stimmt und darin liegt die Schwierigkeit (und auch der Reiz) einer Evaluation von Rio.

Rio war ein Erfolg weil rund 180 nationale Delegationen und 128 Staats- und Regierungschefs das komplexe, herausfordernde Thema Umwelt und Entwicklung zuoberst auf ihrer Agenda hatten und sicher teilweise weiterhin haben. Drei Konventionen wurden unterzeichnet: über Klima, Biodiversität und Wälder. Dievon der Konferenz lancierte Erklärung von Rio enthält klare Aussagen über die wesentlichen Grundsätze einer nachhaltigen Entwicklung. Schliesslich wurde ein rund 900-seitiges Sammelwerk von Ideen und Anregungen für zukünftiges Handeln als Agenda 21 verabschiedet. Die rund 9'000 Journalisten informierten und sensibilisierten die Weltöffentlichkeit vor, während und nach der Konferenz. Die Teilnahme von Hunderten von Nicht-Regierungsorganisationen (NGOs) und von Vertretern der Unternehmerschaft war ein für die UNO und für die Welt erstmaliges Ereignis, das ein weites Feld für zukünftige Zusammenarbeit öffnete.

* Cf. résumé p. 262. 
Falls die vielen Unterschriften unter den verschiedenen Dokumenten dazu führt, dass den eingegangenen Verpflichtungen auch nachgelebt wird, würde dies die Welt nach Rio tatsächlich ändern. Dann würden das Vorsorgeprinzip und das PPP-Prinzip (Polluter-Pays-Principle) breite Anwendung finden; dann würden marktwirtschaftlich orientierte Instrumente der Umweltpolitik vermehrt eingesetzt; dann würden sich die Leistungen des Nordens für die Entwicklungszusammenarbeit verdoppeln; dann würde Technologiekooperation kein leeres Wort bleiben, sondern zu einem eigentlichen Innovations- und Diffusionsprogramm in Entwicklungsländern führen; dann würde sich die internationale Zusammenarbeit zwischen den Staaten verbessern; dann würden private Initiativen viel Raum für eigenständige Anstrengungen und neuartige Kooperationsformen zwischen NGOs und der Geschäftswelt finden.

Die grosse Frage lautet demnach: Wieviel gelten diese Unterschriften? Wie ernsthaft sind die Vorschläge in Agenda 21 gemeint? Wieviel guter Wille wird den guten Worten folgen? Niemand kennt die Antworten auf diese Fragen, da sie sich erst im laufenden und zukünftigen politischen Prozess herausbilden werden. In demokratischen Ländern sind es letztlich die Bürgerinnen und Bürger, die mit Wahl- und Sachentscheidungen die Qualität der Umsetzung der eingegangenen Verpflichtungen bestimmen werden.

Rio war aber auch ein Misserfolg, denn selbst bei optimistischer Einstellung muss im Sinne einer kritischen Evaluation der Konferenz auf vier schwerwiegende Lücken hingewiesen werden:

- Die UNCED war eine Umwelt-, aber keine Entwicklungskonferenz. Die reicheren Länder dominierten die Thematik (vor allem Klima, Biodiversität, Wald), während die - anfangs sehr skeptisch eingestellten - Entwicklungsländer ihre Chance zu spät oder gar nicht wahrnahmen. Wir wissen, nicht zuletzt dank UNCED, dass die Wirtschaftsgeschichte der Industrieländer nicht einfach wiederholbar ist. Trotz UNCED sind jedoch nur wenige, verschwommene Ideen zum neuen Entwicklungsweg sichtbar, der wirtschaftliches Wachstum, soziale Chancenvielfalt und ökologisches Gleichgewicht vereinen soll.

- Überzeugende Analysen im Zusammenhang mit Entwicklung, Umweltproblemen und Bevölkerungswachstum wurden vorgelegt, aber keine schlüssigen Folgerungen daraus abgeleitet. Mit Erfolg widersetzte sich insbesondere der Vatikan jeder klaren Empfehlung zur Eindämmung der Bevölkerungsexplosion. Viele Tabus verstellen den Weg zu offensichtlich notwendigen Lösungen. UNCED hat dies nicht zu ändern vermocht.

- Wenig Bewegung kam ins Energiethema. Der harte Widerstand der erdölproduzierenden Staaten und der USA hat weitreichende Vorschläge stark verwässert und zum Teil abgeblockt. Im Gegensatz dazu hat der 15. Weltenergiekongress vom Oktober 1992 wesentlich kräftigere Folgerungen aus dem treffend analysierten Zusammenhang Unterentwicklung-Bevölkerungswachstum-Energiebedarf gezogen (2). 
- Die hohen Erwartungen der Entwicklungsländer im Finanzbereich wurden herb enttäuscht. Das UNCED-Sekretariat hatte auf der Basis von Agenda 21 überhöhte Erwartungen geweckt, die von der Konferenz nicht erfült wurden. Das Versprechen der Industrieländer zur Erhöhung der Entwicklungshilfe auf $0,7 \%$ ihres BSP ist eine Wiederholung früherer Verpflichtungen, deren Erfüllung aufgrund der weltwirtschaftlichen Rezession ausserordentlich schwierig werden dürfte. UNCED hat auch keine neuen Finanzierungswege aufgezeichnet, weil die Debatte kaum vom Ansatz der traditionellen Entwicklungshilfe wegkam.

Die genannten vier Aspekte sind von grosser Bedeutung. Sie begründen hinreichend, warum UNCED kein voller Erfolg war. Allerdings hat die Konferenz wesentliche Grundlagen und Prinzipien erarbeitet, die nachhaltige Wirkungen entfalten dürften. UNCED ist in diesem Sinne ein wichtiger Meilenstein im Prozess der nachhaltigen Entwicklung auf unserer Erde, aber wir stehen offensichtlich erst am Anfang eines langen und hindernisreichen Weges.

\section{Die Essenz nachhaltiger Entwicklung}

Die UNCED 1992 hat zweifellos zur Konkretisierung der Vision von nachhaltiger Entwicklung Wesentliches beigetragen. Dies ist wichtig, weil die Gefahr bestand (und weiterhin in abgeschwächter Weise besteht), dass dieser Schlüsselbegriff verwässert und letztlich unbenutzbar wird (3).

Die drei grundlegenden Erkenntnisse aus der UNCED-Debatte sind:

- Nachhaltige Entwicklung ist ein Prozess, der die Optimierung von beständigem wirtschaftlichem Wachstum, sozialer Chancenvielfalt und ökologischem Gleichgewicht auf möglichst selbstbestimmte Weise anstrebt.

- Nachhaltige Entwicklung wendet sich gegen eine "Politik der ungedeckten Wechsel": Die gegenwärtige Nutzung von natürlichen Ressourcen und Energie soll nicht zu Lasten zukünftiger Generationen und ebensowenig zu Lasten anderer Regionen oder bestimmter sozialer Gruppen geschehen. Auftretende negative externe Effekte sollen konsequent internalisiert werden, weil nur so der Verursacher effektiv zahlt. Dies ist eine zentrale Voraussetzung für nötige Verhaltensänderungen von Konsumenten und Produzenten (4).

- Auf der mikroökonomischen Ebene der Unternehmungen bedeutet nachhaltige Entwicklung eine Kursänderung in Richtung Öko-Effizienz. Effizienzsteigerungen dank besserer Technologie, Organisation und modernem Ressourcenmanagement wirken sich ökonomisch und ökologisch positiv aus. Wettbewerbsvorteile sind die Folge; sie bilden das entscheidende Argument, um zwischen den Antipoden Wirtschaft und Umwelt eine Brücke bilden zu können.

Diese Erkenntnisse bedeuten einen nicht zu unterschätzenden Wendepunkt in der entwicklungspolitischen Debatte. Das Konzept nachhaltiger Entwicklung ist 
umfassend: Produzenten, Arbeitnehmer, Konsumenten und Politiker werden gleichermassen angesprochen; alle Lebensbereiche sind integriert; die Gegensätze und Zusammenhänge zwischen Reich und Arm werden ins Zentrum gerückt; von der Makroebene weltweiter Thematik bis zur Mikroebene einzelner Akteure sind alle Stufen vertreten; die langfristige Vision wird mit kurz- und mittelfristiger Programmatik ergänzt.

Nachhaltige Entwicklung will das Verhalten von Unternehmern, Konsumenten und Politikern wesentlich beeinflussen. Dies kann nur dann geschehen, wenn vom Eigeninteresse aller Akteure ausgegangen wird. Nötig ist eine grundlegende Ånderung der Rahmenbedingungen, die letztlich Anreize und Sanktionen definieren. So ist das Konzept der Versuch, unser wirtschaftliches und politisches System zugunsten der Prinzipien nachhaltiger Entwicklung zu modifizieren. Eine Neudefinition der institutionell-legalen Rahmenbedingungen, der Subventionen und Steuersysteme, der Nutzungsrechte und Ausführungspflichten ist für die Umsetzung des Konzepts unabdingbar (5).

Eine solche Systemänderung wird unter den Akteuren Gewinner und Verlierer hervorbringen. Insgesamt wird der Systemwandel aber das wirtschaftliche, soziale und ökologische Gesamtresultat nachhaltig verbessern. Die entscheidende politökonomische Frage ist deshalb, inwiefern es im Interesse dieses Fortschritts auf der Makroebene gelingen mag, die potentiellen Gewinner auf der Mikroebene zu motivieren und die potentiellen Verlierer zu neutralisieren. Erhebliche "vested interests" stehen diesem Prozess im Weg. Der notwendige Wandel wird zusätzlich gebremst durch die inhärente Schwerfälligkeit der meisten staatlichen und vieler privater Institutionen (6). Auf verschiedene wichtige Fragen gibt es zudem noch keine befriedigenden wissenschaftlichen Antworten (7) - ein weiterer Grund für eine erhebliche Abneigung vieler Akteure gegen den notwendigen Kurswechsel.

\section{Nachhaltigkeit zwischen Effizienz und Partizipation}

Wenn ein Unternehmen Verluste erleidet, die das Eigenkapital und die Substanz aufzehren, macht es Konkurs. Gewinne sind deshalb eine Voraussetzung für das Überleben, für Investitionen in neue Technologien, Produkte und Märkte, das heisst für die unternehmerische "Nachhaltigkeit".

Diese offensichtliche Erkenntnis hat sich leider in der Politik - und auch in der Entwicklungspolitik - nicht durchgesetzt. Nach wie vor dominiert hier die Meinung, dass das Thema Umwelt und Entwicklung mit einer Mischung von sozialer Verteilungs- und regulativer Umweltpolitik anzugehen ist (8). Dabei wird der "archimedische Punkt" für marktwirtschaftliche Demokratien verfehlt: grundsätzlich streben alle Akteure ihre individuelle Existenzsicherung und Nutzenmaximierung an. Verhaltensänderung in Richtung einer kollektiven Zielsetzung wie nachhaltiger Entwicklung geschieht erst dann, wenn es sich auch für Konsumenten, Produzenten und Politiker lohnt.

Eine wichtige Erkenntnis erhält zunehmende Zustimmung: Öko-Effizienz (9) ist mit offenen Märkten besser erreichbar, da sich durch Wettbewerb effizientere, 
produktivere und innovativere Lösungen durchsetzen. Weitgehend geschlossene Märkte tendieren zu Ineffizienz, Verschwendung von natürlichen Ressourcen und von Energie und damit zu weitverbreiteter "Unsustainability" (10). Osteuropa, Brasilien, Indien sind nicht nur krasse, sondern auch weltweit bedeutende Beweise für diesen Zusammenhang.

Offene Märkte sind auch eine wichtige Voraussetzung, dass mehr Menschen sich am Wirtschaftsprozess beteiligen, mehr und vielfältigere Gelegenheiten sich anbieten, zahlreichere Initiativen eine grössere Chance erhalten. Fähige Kleinunternehmer beispielsweise können ihre Ideen besser umsetzen, NGOs erhalten Zugang zu wichtigen Projekten, Forscher können ihre Innovationsfähigkeit besser nutzen (11).

Mehr wirtschaftliche und politische Partizipation, verbunden mit mehr Effizienz dank offener und freier Märkte, bildet das zentrale Paradigma für nachhaltige Entwicklung. Wichtig ist dabei die Erkenntnis, dass offene Märkte nicht archaische oder völlig ungeordnete Märkte sind, im Gegenteil: offene Märkte benötigen klare Spielregeln, beispielsweise bezüglich Eigentumsrechte, Informationstransparenz, Handelsrecht (12). Erst klare politische Rahmenbedingungen bringen hohe Verlässlichkeit und konsequente Durchsetziungskraft, sie sind deshalb eine notwendige (wenn auch nicht hinreichende) Voraussetzung für nachhaltige Entwicklung.

\section{Chance für die Schweiz?}

Die Schweiz scheint im Schatten ihrer innenpolitischen Probleme zu vergessen, dass sie im weltweiten Vergleich erhebliche Vorteile für den Prozess nachhaltiger Entwicklung besitzt: Die thematische Sensibilisierung ist in der Schweiz weit fortgeschritten, ihre Neutralität bedeutet einen Vertrauensbonus in den meisten Entwicklungsländern, ihre Tradition der guten internationalen Dienste könnte sich ebenfalls positiv auswirken.

Erste Voraussetzung für die Wahrnehmung dieser Chance ist, dass das eigene Haus in Ordnung gebracht wird. Die schweizerische Wirtschafts- und Umweltpolitik benötigt weitgehende Anpassungen, besonders wichtig sind dabei eine grundsätzliche Reform des Steuersystems und die Entwicklung neuer Impulse für die Forschung. Einige aktuelle Untersuchungen (13) zeigen deutlich auf, dass wichtige Marktmechanismen in der Schweiz gestört sind. Viele Preise signalisieren weder die ökonomische noch die ökologische Wahrheit. Bisher hat die Politik darauf mit zusätzlichem wirtschaftspolitischem und umweltpolitischem Interventionismus reagiert. Genau das Gegenteil wäre aber nötig: eine Durchforstung des Subventions-Dschungels, ein Abbau von Regulierungen und eine wesentlich marktorientiertere (statt kontrollorierte) Umweltpolitik.

Die Schweiz steht diesbezüglich nicht allein da. Mehr und nicht weniger Markt ist in ganz Europa notwendig. Eine gezielte Harmonisierung im europäischen Rahmen ist notwendig, um anhaltende Erfolge einer modernen Umweltpolitik (14) zu erzielen. 
Im Bereich der schweizerischen Entwicklungspolitik erfordert das Konzept der nachhaltigen Entwicklung eine grundsätzliche Neuorientierung. Diese reicht weit über die Einführung ökologischer Kriterien in der Projektselektion und -begleitung hinaus (15). Wichtigste Überlegungen sind:

\section{- Klare Zielsetzung:}

Die schweizerische Entwicklungszusammenarbeit muss konsequenter auf die drei zentralen Ziele von nachhaltiger Entwicklung in Entwicklungsländern ausgerichtet werden:

- wirtschaftliches Wachstum

- soziale Chancenvielfalt und

- ökologisches Gleichgewicht.

.Bisher wurde das - im Gesetz auch besonders hervorgehobene - Ziel der sozialen Gerechtigkeit gegenüber den wirtschaftlichen und ökologischen Anforderungen stark betont. Ein besseres Gleichgewicht ist nötig.

\section{- Besserer Mitteleinsatz:}

Die Qualität und Effizienz des Mitteleinsatzes sind gegenwärtig nicht optimal. Die heutige Organisation der schweizerischen Entwicklungszusammenarbeit ist charakterisiert durch extreme Dezentralisierung ohne genügende Koordination. Wir wissen wenig über die Gesamtwirkungen der schweizerischen Entwicklungspolitik. Es bestehen zwar Evaluationsberichte zu einzelnen Projekten und Programmen sowie zu einzelnen Instrumenten wie dem Mischkredit. Eine konzeptionelle Gesamtbeurteilung bezüglich Zielerreichung, Effektivität und Effizienz steht jedoch noch aus. Sie ist dringend nötig, um eine seriöse Ausgangslage für die zukünftige Gestaltung der Entwicklungspolitik zu schaffen.

\section{- Stärkere Kohärenz:}

Die privaten Investitions- und Handelsbeziehungen der Schweiz mit den Entwicklungsländern weisen wesentlich höhere Zahlen als jene für die Entwicklungszusammenarbeit aus; vor deren weiterem Ausbau müsste deshalb das aussenwirtschaftliche Instrumentarium durchforstet werden. Steht die Schweiz zu ihren Freihandelsprinzipien auch im Verhältnis zu den Entwicklungsländern? Setzt sie sich im GATT für entsprechende Reformen ein ? Klar ist beispielsweise, dass der Schutz der Landwirtschaft - mit rund sechsmal höheren Aufwendungen pro Kopf als die gesamte Entwicklungszusammenarbeit - unsere Entwicklungspolitik untergräbt. Eine faire, weniger protektionistische Handelspraxis wäre wahrscheinlich wesentlich wirksamer als zusätzliche Mittel für Entwicklungshilfe. 


\section{- Mehr Chancenvielfalt dank besseren Rahmenbedingungen:}

Nachhaltige Entwicklung benötigt wirtschaftliches Wachstum durch möglichst vielfältige Initiativen in möglichst vielen Händen. An Talenten und Risikobereitschaft fehlt es in der Mehrzahl der Entwicklungsländer nicht. Der Zugang zu den Märkten ist schwierig, der Einsatz von Produktionsfaktoren wie Kapital oder Technologie ist kompliziert und teuer, es bestehen besonders grosse Hindernisse bezüglich Registrierung und Inwertsetzung von Eigentum. Mehr Wachstum durch mehr private Initiativen ist wirtschaftlich, sozial und politisch von grösster strategischer Bedeutung. Dies setzt offene Märkte voraus und verlangt privatwirtschaftliches Engagement für eine tragende Partnerschaft mit Kleinunternehmern. Offene Märkte und wirtschaftliche Wettbewerbsfähigkeit, transparente und für alle gültige institutionelle und rechtliche Spielregeln sowie demokratischere Entscheidungen und Kontrollen lauten die Prinzipien für das nächste Dezennium. Dies erfordert tiefgreifende Veränderungen der Rahmenbedingungen in den meisten Entwicklungsländern. Mit den kargen Ressourcen der schweizerischen Entwicklungszusammenarbeit sollten Projekte nurmehr dort gefördert werden, wo echte Chancen für einen nachhaltigen Kurswechsel bestehen.

\section{- Eigene Stärken nutzen:}

Die thematische und geographische Vielfalt der schweizerischen Entwicklungszusammenarbeit ist gross. Wahrscheinlich gibt es kein Sachgebiet, in welches der Bund nicht direkt oder indirekt Geld investiert. Einer eigentlichen Schwerpunktbildung stehen gewachsene Partnerschaftsverhältnisse und zu diffuse Prioritäten entgegen. Die zunehmende Budgetverlagerung auf Hilfswerke dürfte diese Vielfalt eher noch vergrössern. Folge davon sind zu hohe Begleitkosten und zu wenig anwendungsorientierte Erfahrungskumulation. Eine stärkere Konzentration auf weniger, strategisch wichtige Themen würde Effektivität und Effizienz des Mitteleinsatzes erhöhen.

$\mathrm{Zu}$ überprüfen ist in diesem Zusammenhang, ob die Schweiz wirklich ihre Trümpfe zugunsten einer effektiven Entwicklungspolitik ausspielt. Zweifel sind angebracht. Mit erheblichem Aufwand, engagiert sich die Schweiz bilateral beispielsweise für tropische Landwirtschaft, vermittelt aber praktisch keine Unterstützung im Bereich staatspolitischer, institutioneller und legaler Rahmenbedingungen, wo sie tatsächlich über beträchtliches eigenes Know-how verfügt. Weitere "centers of excellence" liegen weitgehend brach, beispielsweise Aus- und Weiterbildung von Lehrerinnen und Lehrern, Berufsbildung, Umweltpolitik, Kleinunternehmerförderung, Tourismusentwicklung und Reform der Kapitalmärkte. Die genannten Themen sind alle von erstrangiger Bedeutung für die Entwicklungsländer. Diese "centers of need" warten offensichtlich bisher meist vergeblich auf kompetente Partner.

Diese wenigen Hinweise für eine künftige Ausgestaltung der schweizerischen Wirtschafts-, Umwelt- und Entwicklungspolitik sind prinzipieller und strategischer Art. Ein grundsätzliches Überdenken erscheint vielversprechend. Dies setzt aller- 
dings Offenheit in der Analyse, Transparenz in der Evaluation der bisherigen Politik und Mut zur Beseitigung von Tabus voraus. Die Erreichung des Ziels einer nachhaltigen Entwicklung setzt somit nicht nur wesentlich mehr Einsatz von Wissen und Ressourcen voraus, sondern einen ausgesprochen starken Willen und grosse Durchsetzungskraft.

\section{Anmerkungen}

1) Interview mit Maurice Strong in Financial Times vom 14.8.1992, deutsche Übersetzung Ernst A. Brugger.

2) NZZ Nr. 226 vom 29.9.1992, Seite 37.

3) Der Begriff "sustainable development" wurde vor rund 20 Jahren in Kreisen der IUCN (The World Conservation Union) und des WWF geprägt, aber erst durch den Bericht der Brundtland-Kommission weltweit bekannt (vgl. Our Common Future, The World Commission on Environment and Development, Oxford University Press, Oxford \& New York, 1987).

4) Zu dieser Diskussion im Detail: Ernst A. Brugger/Salome Steib im Bericht Umweltpolitik aus unternehmerischer Sicht, hrsg. vom Bundesamt für Konjunkturfragen, Bern, 1992.

5) Eine umfassende Darstellung dieses Gedankenganges bietet Stephan Schmidheiny in Kurswechsel, Artemis \& Winkler Verlag, Zürich, 1992.

6) Siehe den Beitrag von Ernst von Weizsäcker in Ökologische Steuerreform, Samuel P. Mauch/Rolf Iten/Ernst U.v.Weizsäcker/Jochen Jesinghaus, Verlag Rüegger Zürich, 1992.

7) Interessant ist in diesem Zusammenhang: William R.Cline, Global Warming: The Economic Stakes, Institute for International Economics, Washington 1992.

8) Ernst von Weizsäcker zeichnet in seinem Buch Erdpolitik (Wissenschaftliche Buchgesellschaft, Darmstadt, 3. Auflage 1992) nach, dass diese traditionelle Sichtweise nicht "sustainable" ist.

9) Der Begriff "Öko-Effizienz" wird u.a. definiert und argumentiert in: Ernst A. Brugger, Eco Eficiencia, Oveja Negra, Bogotá 1992.

10) Zur Gegenüberstellung offener und geschlossener Systeme, vgl. Mikhail S. Bernstam, The Wealth of Nations and the Environment, occasional paper 85, The Institute of Economic Affairs, London, 1991.

11) Für Entwicklungsländer hat dies Hernando de Soto eindrücklich dargestellt: Marktwirtschaft von unten, Orell Füssli Verlag, Zürich, 1992

12) H. de Soto/St. Schmidheiny, Las otras reglas del juego, Oveja Negra, Bogotá, 1991.

13) Hiezu auch Bruno Fritsch, Mensch-Umwelt-Wissen, Teubner Stuttgart, 3. Auflage, 1992.

14) Es ist deshalb wichtig, die Konsequenz der EG für die schweizerische Umweltpolitik zu analysieren. Vgl. Ecosens/IPSO/BHP-Bericht, Europäische Integration \& Ökologische Folgen für die Schweiz, hrsg. von BUWAL, Bern, Juni 1992.

15) Weiterführende Literatur:

- Richard Gerster, Aus Fehlern lernen?, Ex Libris Verlag, Zürich, 1987;

- Ernst A. Brugger, Artikel Die Schweiz und die Entwicklungsländer, NZZ 23.9.91 